\title{
RESEÑA DE CUADROS GARRIDO, M.E. Y SELMA PENALVA, A. (DIRS.) Y RÍOS MESTRE, J.M. (COORD.) (2021). TÉCNICAS DE INNOVACIÓN DOCENTE EN DERECHO DEL TRABAJO Y DE LA SEGURIDAD SOCIAL. CIZUR MENOR (NAVARRA): ARANZADI, 316 PÁGS.
}

\author{
Alejandro Muros Polo* \\ Universidad de Granada, España
}

Los nuevos postulados del Espacio Europeo de Educación Superior sitúan al alumno en el centro de su propio proceso de enseñanza-aprendizaje. En efecto, el rol del profesor universitario se sitúa ahora en la facilitación de las herramientas de aprendizaje y en el desarrollo de las habilidades y competencias del discente, para que este pueda "aprender a aprender» durante toda la vida. En esta línea del aprendizaje por competencias, resulta obligado el recurso a metodologías docentes activas e innovadoras, sobre todo con ayuda de las TIC en un modelo híbrido o de blended learning.

La transformación digital que ya se está viviendo, también conocida como Cuarta Revolución Industrial, va a demandar para los futuros profesionales y empleos del mańana, no solo competencias digitales, sino también capacidades de comunicación oral y escrita, pensamiento abstracto, solución creativa a los problemas, así como las llamadas habilidades blandas o soft skills. El profesor universitario, por tanto, no puede hacer caso omiso a esta nueva realidad social y profesional, sobre todo si se quiere mejorar la empleabilidad de los egresados de la educación superior, ya muy dañada por la alta tasa española de desempleo juvenil.

\footnotetext{
* Correspondencia a: Alejandro Muros Polo. Plaza de la Universidad, n. 1 (18071-Granada), España. — amurospolo@ugr. es - https: //orcid.org/0000-0002-6192-8481

Cómo citar: Muros Polo, Alejandro. (2021). "Reseña de Cuadros Garrido, M.E. y Selma Penalva, A. (dirs.) y Ríos Mestre, J.M. (coord.) (2021). Técnicas de innovación docente en Derecho del Trabajo y de la Seguridad Social. Cizur Menor (Navarra): Aranzadi, 316 págs.n; Lan Harremanak, 46, 203-206. (https: //doi.org/10.1387/lan-harremanak.23174).

Recibido: 11 noviembre, 2021; aceptado: 13 noviembre, 2021.

ISSN 1575-7048 - elSSN 2444-5819 / (C) 2021 UPV/EHU
}

(c) Esta obra está bajo una licencia

Creative Commons Atribución 4.0 Internacional 
Buena prueba de la docencia a distancia o semipresencial basada en las tecnologías de la información y la comunicación ha sido la experiencia vivida a causa de la COVID-19, especialmente a través del campus virtual y las plataformas de videollamada. Así, con esta experiencia previa se han podido comprobar tanto las ventajas como los inconvenientes del e-learning y del uso de nuevas tecnologías en una disciplina de tanta tradición, como es la del Derecho y, en concreto, en su rama del Derecho del Trabajo y de la Seguridad Social. Entre las ventajas destaca la importancia de incorporar metodologías activas de aprendizaje, en la línea de aportar mayor dinamicidad y agilidad en el desarrollo de competencias, así como para facilitar la aplicación práctica de los contenidos teóricos ante situaciones reales que se puedan dar en la práctica profesional. Y es que se puede llegar a decir que la docencia universitaria ha cambiado con la llegada de la crisis pandémica.

Teniendo en cuenta todo lo anterior, la presente obra colectiva Técnicas de innovación docente en Derecho del Trabajo y de la Seguridad Social, dirigida por las profesoras Cuadros Garrido y Selma Penalva, y coordinada por el profesor Ríos Mestre, todos ellos integrantes de la Universidad de Murcia, se plantea como una obra colectiva de importante actualidad y de necesaria lectura para el docente universitario, habida cuenta de sus valiosas implicaciones prácticas. En efecto, la monografía se dirige a los profesores universitarios, en general, y a los docentes iuslaboralistas, en particular, con el fin de reforzar su formación docente, la cual está adquiriendo cada día más importancia en el currículum y en la promoción del profesorado universitario.

Nos encontramos, por tanto, ante una obra de referencia para la enseñanza del Derecho y de su rama del Derecho Trabajo y de la Seguridad Social, que ha sido elaborada por prestigiosos juristas y profesores universitarios expertos en Derecho laboral, no solo provenientes de diferentes universidades españolas, sino de otros países europeos e, incluso, latinoamericanos. Lo cual ha dotado a la obra colectiva de un carácter internacional, aspecto muy importante en la actual época de globalización. Además, otro aspecto que suma valor a la presente monografía se sitúa en su publicación en la insigne editorial de Aranzadi, referencia en el campo del estudio jurídico de las relaciones laborales.

En cuanto a la estructura de la obra colectiva, la misma se divide en un prólogo, elaborado por el Decano de la Facultad de Derecho de la Universidad de Murcia, Don David Morillas Fernández, seguido de cinco partes respectivamente dedicadas a:

— «Parte general. Innovación docente en Derecho del Trabajo y de la Seguridad Social». En esta primera parte, se integran tres capítulos que versan sobre «La enseñanza del Derecho del Trabajo con perspectiva de género», elaborado por la profesora Alejandra Selma Penalva, de la Universidad de 
Murcia; «Una propuesta de evaluación continua», elaborado por el profesor José María Miranda Boto, de la Universidad de Santiago de Compostela; $\mathrm{y}$ «La docencia dual como respuesta a la COVID-19 y la enseñanza del Derecho del Trabajo: algunas propuestas pedagógicas para facilitar la interiorización de la disciplina en el nuevo contexto", elaborado por la profesora Carolina Blasco Jover, de la Universidad de Alicante.

- «Técnicas de innovación docente en el contexto actual: aprendizaje colaborativo en la enseñanza on line y semipresencial del Derecho laboral». En esta segunda parte, se integran los siguientes capítulos de libro dedicados a: «El uso de teams en la docencia: ventajas y desventajas», elaborado por la profesora Gratiela Florentina Moraru, de la Universidad de Castilla-La Mancha; "El trabajo en equipo de alumnado aplicado a la docencia práctica de Derecho del Trabajo y de la Seguridad Social», elaborado por la profesora Inmaculada Hernández Morales, de la Universidad de Murcia; «Implementación de las habilidades y acción comunicativa en el Derecho social del Trabajo», elaborado por la profesora Belén del Mar López Insua, de la Universidad de Granada; «Trabajo en grupo y aprendizaje cooperativo: una experiencia docente aplicada al ámbito de la Seguridad Social», elaborado por la profesora Concha Sanz Sáez, de la Universidad de Castilla-La Mancha; «Los objetivos ODS en la docencia de Derecho del Trabajo", elaborado por la profesora M. ${ }^{a}$ Elisa Cuadros Garrido, de la Universidad de Murcia; y «Una experiencia de aprendizaje basado en proyectos aplicada a la prevención de riesgos laborales», elaborado por la profesora Mirentxu Marín Malo, de la Universidad Pública de Navarra.

- «La relevancia de los aspectos tecnológicos de la innovación docente del Derecho Social». En esta tercera parte, se integran los siguientes capítulos: "The teaching labour law and the access to databases of jurisprudence», elaborado por los profesores Frabizio Ferraro y Gianluca Giampá, provenientes de la Universidad de la Sapienza (Italia); "Contactos, pantallazos y blogs: recursos para la docencia en Derecho del Trabajo», elaborado por el profesor Antonio Fernández García, de la Universidad Oberta Catalunya; «Privacy and personal data protection in the virtual teaching-regulations, risks and good practices», elaborado por la profesora Kamila Naumowicz, de la Universidad de Warmia and Mazury (Polonia); «El uso de twitter como herramienta docente», elaborado por el profesor Daniel Pérez del Prado, de la Universidad Carlos III de Madrid.

— «Solución de conflictos y docencia del Derecho del Trabajo». En esta cuarta parte, se integran diferentes capítulos dedicados a: «Apuntes y materiales sobre la formación judicial especializada (social) en España», elaborado por el magistrado especialista en la jurisdicción social y vocal del Consejo General del Poder Judicial, Juan Martínez Moya; «Trabajos fin 
de estudios sobre disciplinas laborales a partir de la colaboración con clínicas jurídicas pro bono", elaborado por la profesora María Salas Porras, de la Universidad de Málaga; e «Innovación docente en la materia de Derecho Procesal Laboral», elaborado por la profesora Yolanda Maneiro Vázquez, de la Universidad de Santiago de Compostela.

- «La vigencia innovadora de los elementos tradicionales de difusión del conocimiento». En esta quinta y última parte de la presente obra colectiva, se integran los siguientes capítulos que versan sobre: «La enseñanza del derecho en el sistema jurídico continental europeo», elaborado por el profesor José María Ríos Mestre, de la Universidad de Murcia; «El cine en el salón virtual, como recurso didáctico para la enseñanza del derecho del trabajo en el mundo post-pandemia», elaborado por la profesora Andrea Isabel Franconi, proveniente de la Universidad de Buenos Aires (Argentina); "Charles Dickens y la obra "Tiempos difíciles» como ejercicio didáctico para la enseñanza del derecho del trabajo», elaborado por el profesor Julen Llorens Espada, de la Universidad Pública de Navarra; y «El empleo del método socrático en el análisis sobre la responsabilidad en el accidente de trabajo», elaborado por el profesor Jesús Martínez Pérez, de la Universidad de Murcia.

En definitiva, la obra colectiva aquí recensionada se muestra como una obra de referencia, tanto por sus autores como por su contenido, que reúne las principales técnicas de innovación docente en el ámbito de la enseñanza y aprendizaje del Derecho del Trabajo y de la Seguridad Social. El Derecho y, especialmente, el Derecho del Trabajo, así como la sociedad y la nueva realidad productiva están en constante cambio, y el profesor universitario no puede quedarse atrás. 